\title{
Kinematics of Initiating a Two-Joint Arm Movement in Patients with Cerebellar Ataxia
}

\author{
Steve Massaquoi and Mark Hallett
}

\begin{abstract}
Objective: To characterize kinematically any systematic aberration in multi-joint movements in cerebellar ataxia. Methods: Nine patients with cerebellar degeneration and nine normal subjects, mobile only at the shoulder and elbow of the right arm, were required to produce left-to-right cross-body linear hand trajectories on the horizontal surface of a digitizing tablet. Nonlinearity indicated failure of precise coordination of the two joints. A wide range of hand speeds was studied. Data analysis was restricted primarily to the first $130 \mathrm{~ms}$ of movement. Results: As hand velocities increased, normal subjects and, especially, patients produced misdirected, curved paths. Normal subjects had significant curvature when peak speeds exceeded $100 \mathrm{~cm} / \mathrm{s}$ and a trend toward significant bi-directional angular deviation at velocities greater than $300 \mathrm{~cm} / \mathrm{s}$. In patients, peak path curvature was significantly greater than normal at peak velocities of 50 to $200 \mathrm{~cm} / \mathrm{s}$. By $3.3 \mathrm{~cm}$, their paths deviated significantly outward at all but the slowest speeds. Overall, patients' maximal hand velocities and shoulder angular velocities, as well as maximal angular accelerations at both joints, were significantly lower than normal. Conclusions: The patients' trajectory aberrations were attributed to a deficient rate of rotation at the shoulder relative to that at the elbow. Relative to task requirements, their rate of torque development was apparently deficient at both joints, but to a greater degree at the shoulder. Joint torque-rate impairment may contribute to the ataxia in both multi- and single-joint movements of patients with cerebellar disorders. A similar, but smaller impairment may produce milder nonlinearity in high-velocity movements of normal subjects.
\end{abstract}

RÉSUMÉ: Cinématique du début d'un mouvement bi-articulaire du membre supérieur chez des patients atteints d'ataxie cérébelleuse. But: Caractériser par la cinématique toute aberration systématique dans les mouvements impliquant plusieurs articulations dans l'ataxie cérébelleuse. Méthodes: Nous avons demandé à neuf patients atteints de dégénérescence cérébelleuse et à neuf sujets normaux, dont la mobilité était limitée à l'épaule et au coude du membre supérieur droit, d'inscrire une trajectoire linéaire de gauche à droite sur la surface horizontale d'une tablette de numérisation placée devant eux. L'incoordination des deux articulations était révélée par toute déviation du tracé. Nous avons étudié une gamme étendue de vitesses de la main et analysé les données des premières $130 \mathrm{~ms}$ du mouvement. Résultats: À mesure que la vitesse de la main augmentait, les sujets normaux et surtout les patients inscrivaient des tracés curvilignes mal dirigés. Les tracés des sujets normaux étaient significativement incurvés quand la vitesse maximale dépassait $100 \mathrm{~cm} / \mathrm{s}$ et manifestaient une tendance à une déviation angulaire bidirectionnelle significative quand la vitesse dépassait $300 \mathrm{~cm} / \mathrm{s}$. Chez les patients, la courbure maximale du tracé était significativement augmentée par rapport à la normale à des vitesses maximales de 50 à $200 \mathrm{~cm} / \mathrm{s}$. A $3.3 \mathrm{~cm}$, le tracé déviait significativement vers l'extérieur, sauf aux vitesses inférieures. Dans l'ensemble, les vitesses maximales de la main chez les patients et les vitesses angulaires de l'épaule de même que les accélérations angulaires maximales aux deux articulations étaient significativement plus faibles que la normale. Conclusion: Les aberrations de la trajectoire inscrite par les patients ont été attribuées à une vitesse de rotation plus lente à l'épaule qu'au coude. Pour les exigences de la tâche à accomplir, le taux de développement du moment de torsion était déficient aux deux articulations, mais surtout au niveau de l'épaule. Un déficit du taux du moment de torsion articulaire peut contribuer à l'ataxie dans les mouvements impliquant une ou plusieurs articulations chez les patients atteints de désordres cérébelleux. Une atteinte semblable mais moindre peut engendrer une non linéarité dans les mouvements de haute vélocité chez les sujets normaux.

Can. J. Neurol. Sci. 1996; 23: 3-14

Ataxia, the incoordination that causes inaccuracy in voluntary movements, is a central clinical feature of cerebellar disorders. Assuming that cerebellar patients begin with normal spatial perception of a target, an assumption supported by observations in monkeys, ' their failure to reach the target with accuracy must be due to improper formulation or execution of the motor plan. It is known that simple, single-joint limb movements are disordered in cerebellar patients. ${ }^{2-7}$ In particular, pack- aging of the EMG bursts is disordered during targeted ballistic movements. ${ }^{4,5.7}$ Bursts in the agonist muscles, for example, may

From the Human Motor Control Section, Medical Neurology Branch, National Institute of Neurological Disorders and Stroke. National Institutes of Health. Bethesda. Maryland.

RECEIVED AUGUST 3, 1994, ACCEPTED IN FINAL FORM ALGUST 21, 1995.

Reprint requests to: Mark Hallett, M.D., Clinical Director, NINDS, NIH, Building 10 Room 5N226, 10 Center Drive MSC 1428, Bethesda. Maryland. USA 20892-1428 
develop slowly and be prolonged, and tend to correspond to hypermetric trajectories. ${ }^{8.9}$

Thach et al. ${ }^{10}$ and Goodkin et al. ${ }^{11}$ argue that the derangements of single-joint movements produced by individual cerebellar nuclear lesions are relatively minor. In monkeys, for example, reaction time delays of 20 to $150 \mathrm{~ms}$ are the chief functional changes. These authors suggest, therefore, that the cerebellum may be preferentially concerned with multi-joint movements. Clearly, though, precise multi-joint movement control may depend critically on proper control of the individual joints active in the movement. Nonlinear dynamics, joint coupling effects, and geometry determine that trajectory errors in multi-joint movements are not simply the sum of, or proportional to, those occurring in isolated movements of the individual joints. Instead, individual joint movement errors may combine synergistically. ${ }^{2}$ In fast multi-joint movements in particular, a delay of only $100 \mathrm{~ms}$ or a slightly reduced rate of acceleration of one joint relative to another may result in a large trajectory aberration. Finally, experiments in which cerebellar nuclei are individually compromised do not necessarily represent the type of functional impairment attending diffuse cerebellar damage. It is conceivable, therefore, that the involvement of the cerebellum in the control of both single-and multi-joint movement is substantial and functionally related.

Cerebellar lesions in humans ${ }^{11,12}$ and animals ${ }^{13.14}$ may result in abnormally large endpoint errors or increased trajectory curvature and variability of rapid, targeted multi-joint arm movements. However, with respect to elucidating the pathophysiology of cerebellar ataxia, the interpretation of results from previous studies is complicated by several factors. First, the analysis of reaching movements performed against gravity must take into account the gravitational influence as an effectively time-varying, trajectory-dependent disturbance force. Second, when the explicit goal is the accurate attainment of a target, and not the production of a particular hand path, different trajectories may be chosen strategically to improve the accuracy." It is possible, therefore, that trajectory variability is not entirely due to involuntary mechanisms. Third, because of the tradeoff in speed and accuracy for targeted movements, ${ }^{15}$ and also possibly because of dynamic effects, aberrations in the trajectory pattern may well be velocity-dependent. Finally, movements lasting many hundreds of milliseconds may involve ongoing conscious correction from visual and proprioceptive feedback. As the cerebellum presumably participates in trajectory correction, as well as in the processing of the original movement command, the net movement potentially reflects the effects of several iterations of signals through abnormal cerebroand spinocerebellar motor control loops. Trajectory errors could become quickly compounded over time in a complicated manner and result in increased trajectory variability. Certainly, visual feedback, or at least simultaneous hand-eye tracking, adversely affects the accuracy of targeting in cerebellar patients. ${ }^{16.17}$ Possibly for some of these reasons, no studies have characterized kinematically any systematic pattern of aberration in multijoint ataxic trajectories.

In an effort to minimize potentially confounding effects and to maximize sensitivity to coordination errors, we selected the task of generating a straight hand path in a cross-body direction horizontally without an end point. Correct performance of the task requires continuously precise control of asynchronous joint motions. Especially if the wrist is immobilized, virtually any aberration in the time course of shoulder or elbow rotation will produce a deviation in the path. Our analysis focused on the first $130 \mathrm{~ms}$ of movement, during which conscious corrections from visual or proprioceptive feedback are minimal. ${ }^{18.19} \mathrm{In}$ an attempt to clarify the importance of movement velocity to trajectory aberrations, we studied a wide range of hand speeds.

\section{Methods}

\section{Subjects}

We studied seven men, aged 29 to 73 years (mean, $52.1 \pm$ 17.1 years), who had severe symptoms of cerebellar cortical atrophy (hereditary type-2, sporadic type-1, alcoholic type-1) or olivopontocerebellar atrophy (hereditary type-2, sporadic type-1); two patients, aged 33 and 34 years, who had mild symptoms of cerebellar cortical atrophy or olivopontocerebellar atrophy (both hereditary type); and nine normal subjects, aged 33 to 80 years (mean, $52.0 \pm 16.5$ years). Subjects were all right-handed. The segregation of the patients into severely and mildly affected groups was made on clinical grounds before the study. There was no clinical evidence of a difference in the quality or severity of ataxia between patients with cerebellar cortical atrophy and those with olivopontocerebellar atrophy, and, therefore, we did not quantitatively explore performance differences between these diagnostic categories. The protocol was approved by the Institutional Review Board, and all subjects gave their written informed consent for the study.

The duration of symptoms ranged from 1 to 30 years. All severely affected patients had evidence of cerebellar hemispheric atrophy, with or without brain stem atrophy, on MRIs or CT scans. Clinically unequivocal ataxia of arm movements was present in all patients, but most prominent in the severely affected ones, who also had ataxia of speech and gait. The patients had no weakness or tremor and no signs of cognitive disturbances or marked abnormalities of the "pyramidal" or "extrapyramidal" systems or of the cranial or peripheral nerves. In particular, patients with clinical findings more severe than extensor plantar responses without upper extremity hyperreflexia or lower extremity clonus, mildly increased tone elicited only through the use of reinforcement maneuvers, or mild proprioceptive disturbance distally in the lower extremities were excluded from the study. Ataxia presenting with radiographic evidence or a family history of cerebellar atrophy in the absence of a marked position sense abnormality constituted clinical evidence of cerebellar dysfunction. None of the patients was taking anticonvulsants, muscle relaxants, anti-Parkinson medication, or psychotropic agents.

The normal subjects were examined and confirmed to be neurologically normal.

Normal subjects and patients, both mildly and severely affected, had comparable mean arm segment lengths. Normal subjects: upper arm, $33.8 \pm 2.54 \mathrm{~cm}$; forearm, $38.9 \pm 2.54 \mathrm{~cm}$. Mildly affected patients: upper arm, $34.3 \pm 1.27 \mathrm{~cm}$; forearm, $40.6 \pm 0.0 \mathrm{~cm}$. Severely affected patients: upper arm, $34.8 \pm$ $1.52 \mathrm{~cm}$; forearm, $39.1 \pm 2.29 \mathrm{~cm}$.

\section{Experimental Procedures}

The subject was seated comfortably in a high-backed chair with his trunk securely immobilized by restraining belts. His 
right arm was free to move across a chest-high, horizontal electromagnetically sensitive surface (Super L II digitizing tablet; GTCO Corp., Columbia, Md.). With the right wrist immobilized by a lightweight splint, the subject firmly held a hard plastictipped electronic stylus in a nearly vertical position, as one would hold a pencil. This arrangement facilitated comfortable access to the digitizing tablet using movements of only the shoulder and elbow. With the stylus in the hand, the arm cleared the table by 5 to $8 \mathrm{~cm}$. The required hand movement, a straight line, was specified by two light-emitting diodes (LEDs); the first indicated the starting position, and the second the direction of the movement (Figure. 1). The subject placed the stylus at the starting position and, any time after the direction-indicator light appeared, moved the stylus toward the light. To achieve maximum velocities, the subject had to avoid stopping at the direction-indicator LED, and therefore was encouraged to continue past the edge of the surface. To suppress any tendency to stop prematurely, the direction-indicator LED was automatically extinguished as soon as movement began. The subject was told explicitly that straightness of the path was more important than the accuracy of direction, and to try not to follow the hand with the eyes, but to look steadily in the direction of the indicator light. The trials were conducted in complete darkness to avoid distractions and visual feedback on hand movement.

Each subject performed two blocks of trials, with 40 movements in each block. So that each block of trials would include a full range of speeds, the subject started out moving "medium fast" and then moved progressively faster until he was moving as fast as possible, and during the last 15 movements slowed

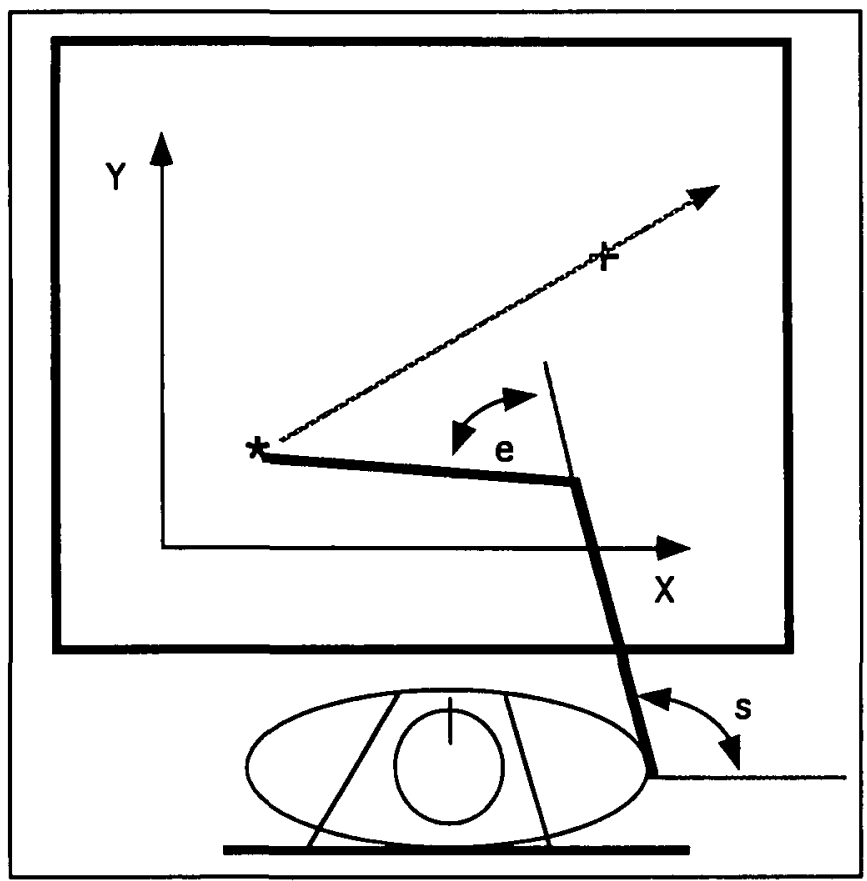

Figure 1: Top view of experimental setup. Subjects used the right arm to produce left-to-right cross-body hand movements. Paths were made from the starting-position LED (*) coordinates $(6.29 \mathrm{~cm}, 5.61 \mathrm{~cm})$ toward the direction-indicator LED $(+)$ coordinates $(41.6 \mathrm{~cm}, 31.1 \mathrm{~cm})$. Trajectories were recorded as coordinate pairs $(X, Y)$, and joint angles at the shoulder (s) and elbow (e) were calculated trigonometrically. progressively. During a 15-minute break between blocks, the subject rested for about 5 minutes before practicing the movements approximately 20 times in the light, using an ink pen on a large piece of paper placed on the tablet surface and marked with the starting and direction-indicator positions. The practice was done to give the subject feedback on his performance and to reinforce the instructions to move in a straight line.

\section{Data Acquisition and Analysis}

The trajectory data were acquired as position coordinates sampled at a frequency of 110 to $130 \mathrm{~Hz}$. The varying frequency is a characteristic of the digitizing tablet that we used. Each acquired point, however, is accurately placed in time. The position data were numerically smoothed using a five-point approximately triangular (low pass) filter with a half-power cutoff of 13 $\mathrm{Hz}$, assuming a fixed sampling interval equal to the mean of the observed sampling intervals (approximately 1/120 s). Smoothed and unsmoothed trajectories were compared visually to verify the absence of shape distortion due to filtering. Differentiation was performed with a five-point differentiating filter based on the same mean sampling interval. The five-point smoothing filter was applied after each differentiation. The beginning of the trajectory was taken as that point at which the tangential (hand) velocity reached $0.5 \mathrm{~cm} / \mathrm{s}$, which was always distinguishable from data "noise." The trajectory shape was quantitatively analyzed by calculating the instantaneous angular deviation from the target direction (direction of direction-indicator LED with respect to starting LED) at checkpoints every $0.76 \mathrm{~cm}$ of arc length (distance along the trajectory) from 0.25 to $7.1 \mathrm{~cm}$. Angular deviations that were counterclockwise from the target direction were outward relative to the body and were defined as positive. The slope of the angular deviation versus arc length curve was calculated between checkpoints. The largest of these values approximates the maximum derivative of direction with respect to arc length, which, by definition, is the peak curvature.

Joint kinematics were quantitatively analyzed in terms of angular movement onset latency, inter-joint interval, initial and peak angular velocity, angular acceleration, and angular jerk. Joint latency is the time after onset of the tangential movement at which the magnitude of the angular velocity in extension exceeded 0.02 radians/s. The inter-joint interval is the difference in time between the onset of shoulder extension and the onset of subsequent elbow extension.

Given trajectory data in Cartesian coordinates, the time courses of joint angular position, angular velocity, angular acceleration, and angular jerk were derived trigonometrically using the measured arm segment lengths and initial shoulder positions for each subject. The differences in the mean initial shoulder coordinates between groups were not significant. A sensitivity analysis indicated that a $1.3-\mathrm{cm}$ error in the measurement of limb segment lengths or of shoulder joint location would yield less than 0.052 radians of angular error and less than 0.2 radians angular velocity error at each joint.

Measured or calculated values for each subject are means derived from the trajectories falling within a specific range of peak hand velocity. "Initial" values are those obtained at the $0.25-\mathrm{cm}$ checkpoint. "Peak" values are the largest ones occurring within the first $130 \mathrm{~ms}$ of a given trial. "Maximal" values are the peak trial values occurring in the highest velocity range achieved by a given subject. 
Regression analysis was used to characterize major trends in the dependence of a subjects' performance on hand speed. For evident physical reasons, several of the measured variables (joint angular velocity, acceleration, and jerk) must equal zero for movements having zero peak velocity. Therefore, all regressions using these data were performed with a zero intercept. With the elbow acceleration and jerk data, the dependence on velocity was substantially linear, so simple linear regressions were performed for each subject. The slope of these regressions measured the steepness of the overall trend in the dependence of the observed variable on peak hand speed. The $t$ test was used to compare the slopes of patients and normal subjects.

For angular velocity and shoulder angular acceleration data, the dependence on velocity appeared to be relatively simple, but not linear. Hence, second-degree polynomial regressions (regressions incorporating both $x$ and $x^{2}$ terms) were used. Each regression, therefore, yielded two coefficients. The first (linear term) coefficient related chiefly to the data's trend at low velocities. The second (nonlinear term) coefficient related chiefly to the data's steepening of rise or tendency toward plateauing at higher velocities. Separate group comparisons using the $t$ test were performed on each type of coefficient. Because there is an increased probability of erroneously finding a significant difference between two groups when multiple $t$ tests are used, a Bonferroni (conservative) correction 20 ( $p$ 116) was applied to adjust the error rates appropriately for multiple (two) comparisons.

The data on angular deviation, peak curvature, and velocity ratio did not have linear or simple nonlinear dependencies on their independent variables. Therefore, multiple $t$ tests and Bonferroni adjustments (four-comparison) were used. With the deviation data, however, because of the inherently high degree of correlation that was likely to exist between the $t$ tests performed at nearby spatial locations, the Bonferroni adjustment was likely to be excessively conservative. In this case, to prevent important data behavior from being obscured, we have reported the results of individual $t$ tests as being "nominally" significant when they attained statistical significance before, but not after the Bonferroni correction. Velocity ratio data were first logtransformed 20 (pp 274-297) and then compared using $t$ tests within each peak hand velocity range followed by Bonferroni adjustments (five-comparison).

Unless otherwise specified, group comparisons were made using two-sample $t$ tests, and statistical significance was defined as $p<0.05$. The results are reported as mean \pm one standard deviation.

Data filtering, trigonometric calculations, graphics, and statistical analysis were done on a Macintosh computer. Both custom-written trajectory analysis and commercial mathematical and statistical software packages were used, including Matlab 3.5 and Statview 4.0.

\section{Results}

\section{Effect of Practice}

Within each velocity range, peak curvature was not significantly different between the first and second blocks of trials in either patients or normal subjects. Angular deviation at $7.1 \mathrm{~cm}$ was significantly reduced between the first and the second blocks of trials in three patients, and there was a trend toward the same reduction in several normal subjects. We interpreted these findings as a practice effect, and, therefore, analyzed only the second, more practiced block of trials for all subjects, on which the following results are based.

\section{Reference Trajectories}

The profiles of tangential (hand) velocity of all movements were rising half-bell-shaped curves. From any given movement, a reference trajectory can be derived by creating artificial data points spaced according to the movement's tangential velocity profile along the correct straight path. From this reference trajectory, "ideal" reference joint time courses can be determined for the given velocity profile.

To obtain more robust reference information, we calculated reference trajectories from the velocity profiles of each of the normal subjects' trajectories. Data from this collection of reference trajectories are presented in parallel with data from the patients and normal subjects as heuristic information. Because of their ad hoc derivation, however, these reference data were not used for statistical comparisons. The trivial exceptions to this were data for peak curvature and angular deviation, where the ideal reference values (zero) are defined absolutely.

\section{Qualitative Analysis of Joint Kinematics}

Typical hand and associated joint paths are shown in Figures 2 and 3. Corresponding joint angular time courses are shown in Figure 4 . The plots show that the kinematic trends that dominated much of the entire movement were already developing within the first $130 \mathrm{~ms}$.

The normal subjects performed well at low and moderate speeds, with hand and joint paths closely approximating ideal behavior. At higher hand speeds, however, they typically showed slight initial elbow flexion, which produced a centripetal component in their hand paths. Elbow extension ensued and became predominant, corresponding to centrifugal hand motion. Together, these motions produced early concavity and subsequent convexity in the Cartesian hand path. Flash ${ }^{21}$ reported a similar sigmoidal morphology of cross-body movements in normal subjects.

In the patients, at moderate speeds, there was no elbow flexion, and elbow extension developed comparatively sooner than in normal subjects. This caused the early, shoulder-dominated portion of the launch to be shorter and the Cartesian hand path to display a concavity that was less deep, though sharper, than in the normal subjects. Some severely affected patients also showed similar abnormal patterns at low speeds.

The high-speed movements of mildly affected patients showed an intermediate pattern with both initial elbow flexion and early elbow extension. Although the patients' trajectory shapes depended on the severity of the ataxia, there was no apparent difference in the shapes regardless of the type of cerebellar atrophy.

\section{Angular Deviation and Path Curvature}

Path deviation differed significantly between patients and normal subjects at certain distances, depending on the peak hand speed. At very slow hand speeds $(<25 \mathrm{~cm} / \mathrm{s})$, the initial angular deviations from the target direction were not significantly different from zero in normal subjects or patients. Although they were 


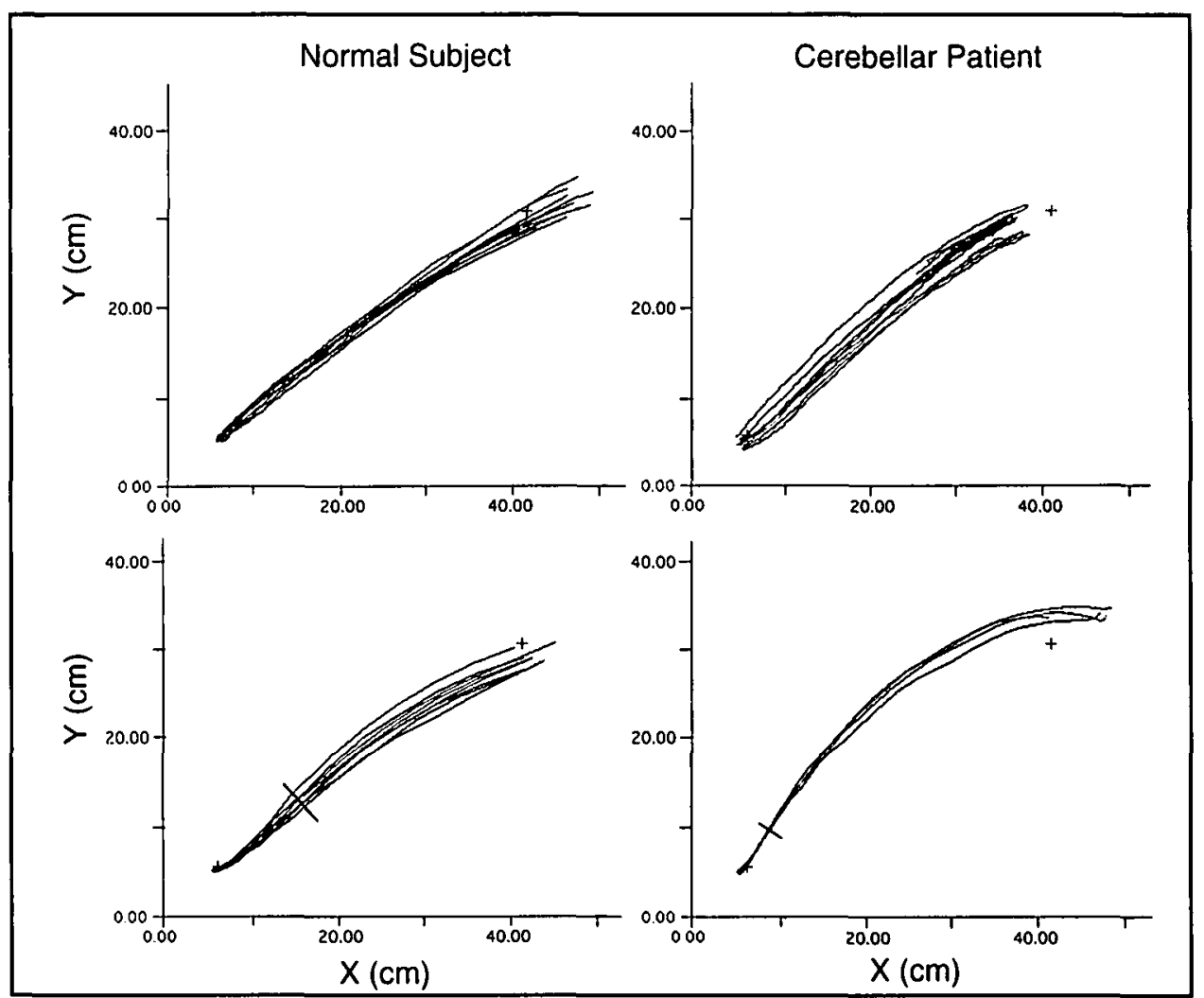

Figure 2: Hand paths produced by a normal subject (left) and a severely affected patient (right) in slower (top, normal subject, 0 - $100 \mathrm{~cm} / \mathrm{s} ;$ patient. $0.50 \mathrm{~cm} / \mathrm{s}$ ) and faster (bottom, normal subject, $250-400 \mathrm{~cm} / \mathrm{s} ;$ patient, 100-200 cm/s) movements. Paths are toward the direction-indicator LED (+). and bars crossing the paths indicate the position at the end of $130 \mathrm{~ms}$ for the fastest of the movements shown.

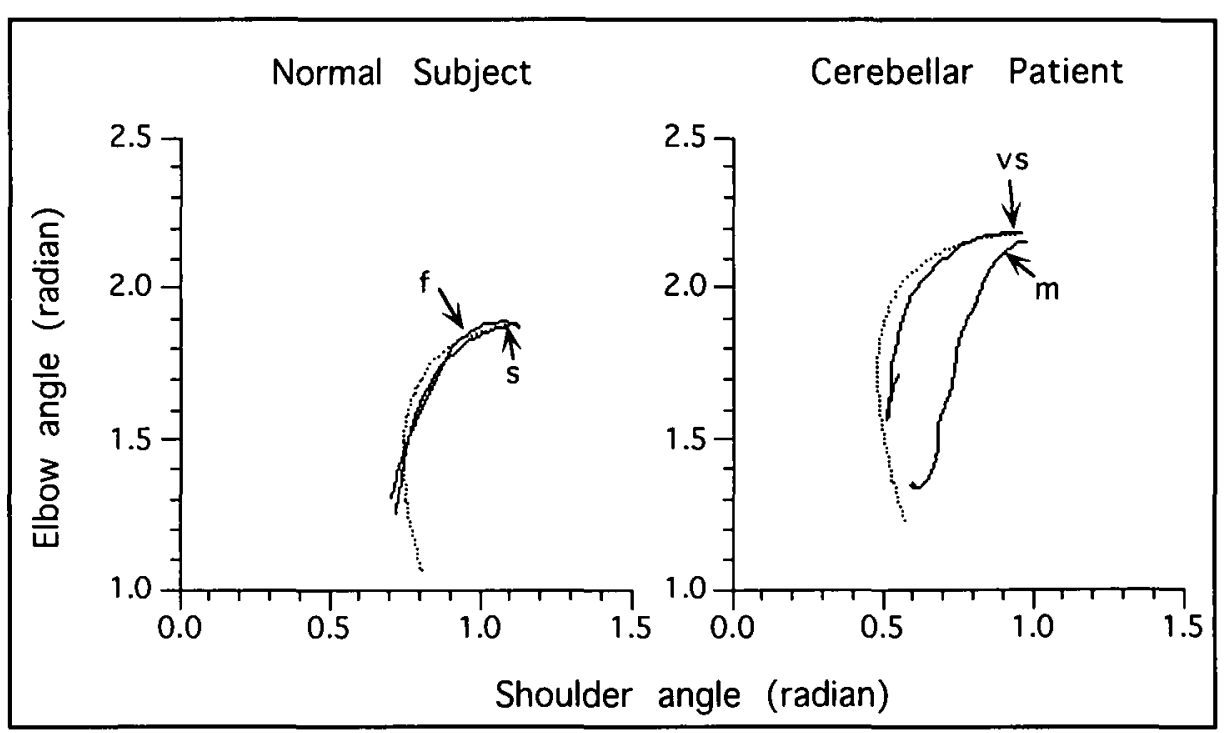

Figure 3: Representative elbow angle versus shoulder angle curves for four trajectories selected from those shown in Figure 2. Trajectories are of very slow $(v s,<25 \mathrm{~cm} / \mathrm{s})$, slow $(\mathrm{s}, 25-50 \mathrm{~cm} / \mathrm{s})$, moderate $(\mathrm{m}, 100-200 \mathrm{~cm} / \mathrm{s})$, and fast $(\mathrm{f}, 300-400 \mathrm{~cm} / \mathrm{s})$ movements in the first $130 \mathrm{~ms}$ of the movement. The reference coordination curve is indicated by the dotted line. Arrows indicate the position at the end of $130 \mathrm{~ms}$. 


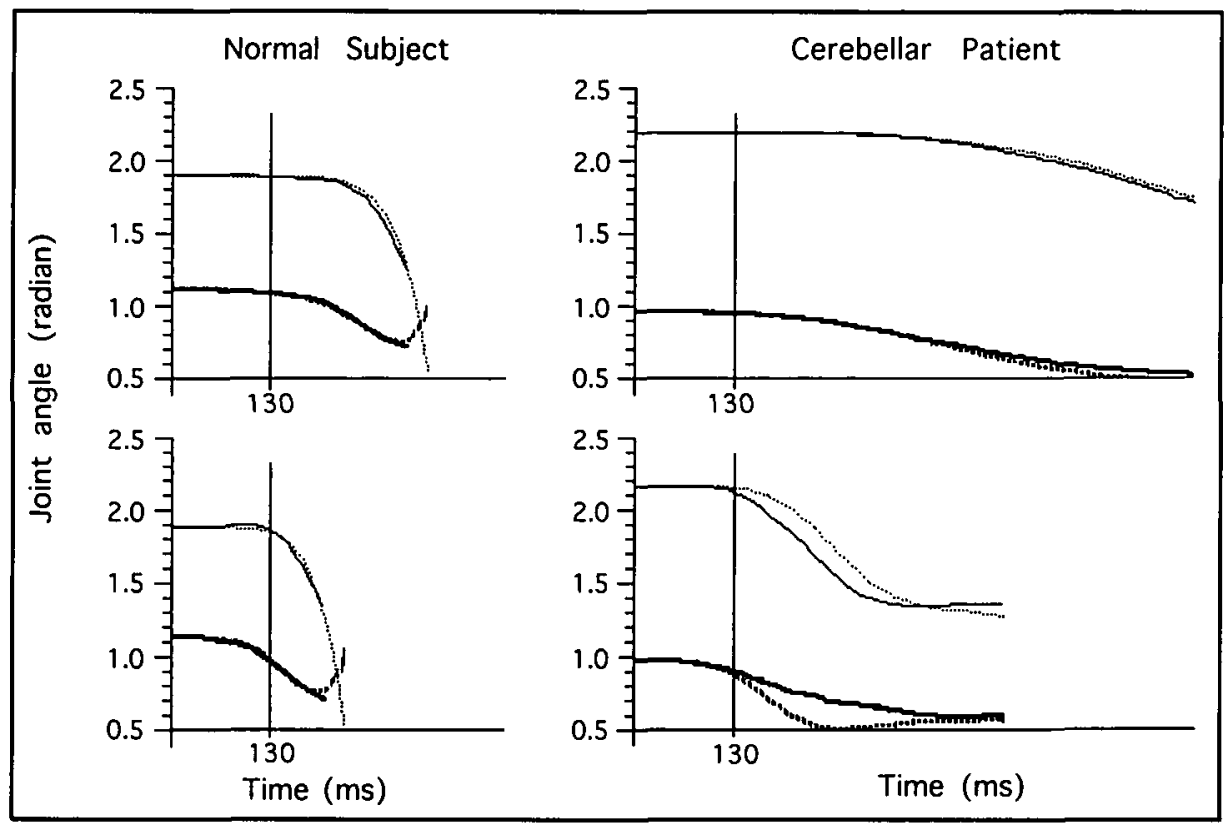

Figure 4: Time courses of shoulder (heavy line) and elbow (light line) rotation for the four trajectories shown in Figure 3. The graphs begin at the time of movement onset. The reference time course of joint motion is indicated by the dotted lines.

not achieved within the 130-ms primary analysis interval, group mean angular deviations at later checkpoints were small $(<0.2$ radian) and not significantly different from each other.

At moderate peak hand speeds $(100-200 \mathrm{~cm} / \mathrm{s})$, all subjects reached at least the 3.3-cm checkpoint. Both normal subjects and severely affected patients had no significant deviations in the initial segment, but severely affected patients showed nominally significant outward deviations at 2.5 and $3.3 \mathrm{~cm}$ (both $p$ $<0.03$ ). The deviation was nominally significantly larger than normal at $2.5 \mathrm{~cm}(\mathrm{p}<0.03)$ and significantly larger than normal at $3.3 \mathrm{~cm}(\mathrm{p}<0.01)$ (Figure 5A). These results are highly consistent with the impression that significant deviation is present in the patients and true differences exist between the patients and the normal subjects at both of these checkpoints. The patterns of deviations at 4.1 and $7.1 \mathrm{~cm}$ are qualitatively similar except that normal subjects began to develop outward deviation as well.

At high peak hand speeds $(300-400 \mathrm{~cm} / \mathrm{s})$, all checkpoints were reached within $130 \mathrm{~ms}$. Normal subjects showed nominally significant deviation inward at $0.25 \mathrm{~cm}(\mathrm{p}<0.05)$ and outward at $7.1 \mathrm{~cm}(\mathrm{p}=0.05)$ (Figure 5B). Generally, the deviations of the two mildly affected patients were comparable to the deviations of normal subjects and severely affected patients when they were similar, and were intermediate when they were significantly different.

Individual paths of some very slow movements had the greatest curvature after $130 \mathrm{~ms}$. For movements faster than $25 \mathrm{~cm} / \mathrm{s}$, however, all peak path curvatures occurred within $130 \mathrm{~ms}$. Overall, the curvature data (Figure 6) indicate that in normal subjects peak path curvature was small at lower velocities, but became significant at higher peak hand velocities. The patients appeared to exhibit substantial path curvature at all velocities, and at moderate velocities, they had significantly more path curvature than normal subjects. Patients, like normal subjects, appeared to exhibit less curvature at lower velocities than at higher ones, but more data are needed to confirm this observation.

\section{Angular Velocity and Angular Velocity Ratio}

Because almost all movement was in extension, which corresponds to negative joint angular velocity, the negatives of the actual values are plotted for ease of interpretation. These plots (Figure 7) suggest that the maximal angular velocities were considerably smaller in severely affected patients than in normal subjects. (Some patients were unable to achieve hand velocities greater than $100 \mathrm{~cm} / \mathrm{s}$, and many, but not all, normal subjects could achieve velocities greater than $400 \mathrm{~cm} / \mathrm{s}$, so group mean maximal performances are not indicated directly by the rightmost data points in Figures 6-8.) Indeed, the group mean maximal velocities at the shoulder and elbow, respectively, were 4.79 $\pm 0.84 \mathrm{radians} / \mathrm{s}$ and $7.33 \pm 2.09 \mathrm{radians} / \mathrm{s}$ for normal subjects and $2.08 \pm 0.61 \mathrm{radians} / \mathrm{s}$ and $2.43 \pm 1.71 \mathrm{radians} / \mathrm{s}$ for severely affected patients. The differences in maximal values between these groups were highly significant at both joints $(\mathrm{p}<0.0001$, shoulder; $p<0.0002$, elbow).

For submaximal hand velocities, peak shoulder angular velocities appeared to rise similarly with peak hand speed in both groups but to plateau earlier in severely affected patients. On the other hand, normal subjects' peak elbow velocities appeared to increase less rapidly than those of severely affected patients as peak hand speeds increased. These impressions were consistent with the regression summaries. For shoulder velocities, the mean nonlinear (second degree) term coefficient was smaller in normal subjects $(0.000025 \pm 0.0000079)$ than in severely affected patients $(0.000041 \pm 0.000020)$ and the mean linear coefficients were comparable. At the elbow, the mean linear coefficient was smaller in normal subjects $(-0.0032 \pm$ 


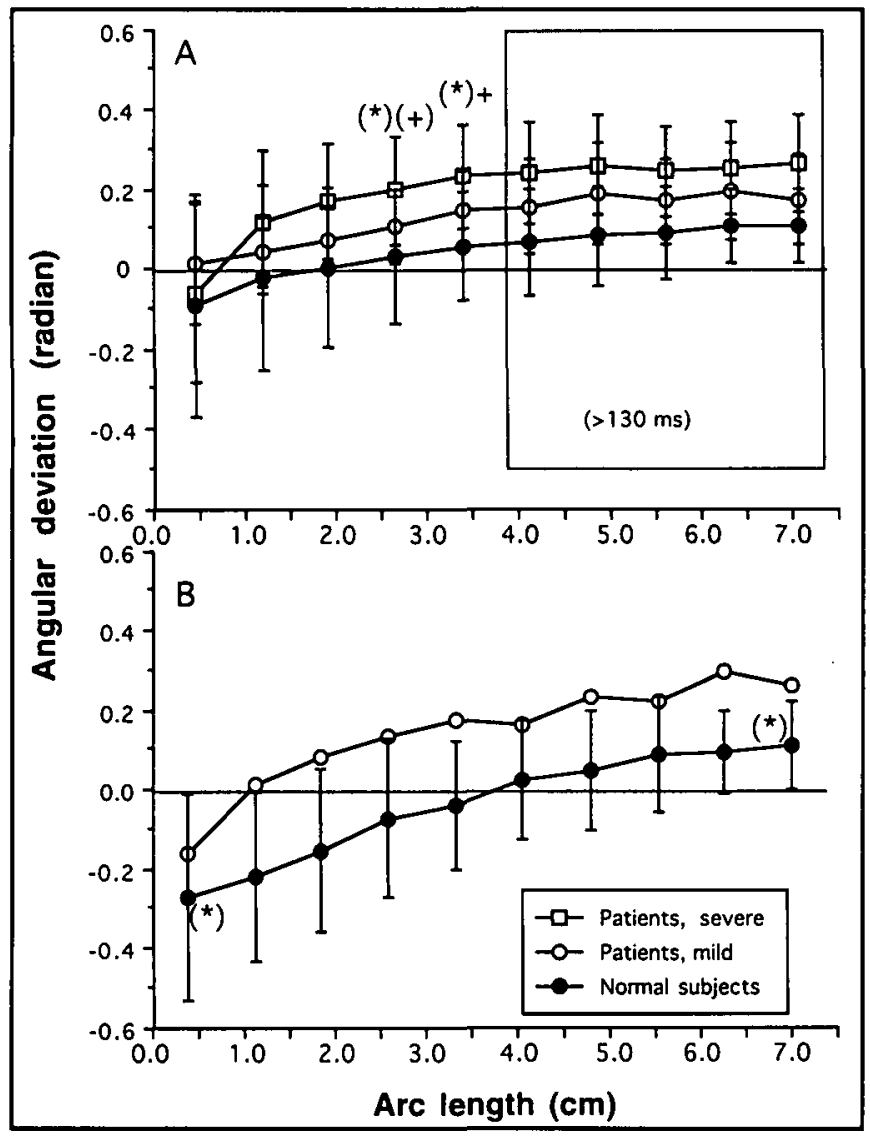

Figure 5: Angular path deviation from instructed direction for $(A)$ moderate-velocity $(100-200 \mathrm{~cm} / \mathrm{s})$ and $(B)$ high-velocity $(300-400 \mathrm{~cm} / \mathrm{s}$ ) movements. Asterisks indicate that the group mean is significantly different from zero. Plus signs indicate that the group mean of patients is significantly different from the group mean of normal subjects. Nominally significant comparisons are indicated by parentheses. Measurements of deviations at arc lengths beyond $3.3 \mathrm{~cm}$ were not made within $130 \mathrm{~ms}$ for some of the (slower) movements in the moderate-velocity range.

$0.0020)$ than in severely affected patients $(-0.0083 \pm 0.0035)$, and the mean nonlinear term coefficients were comparable. These coefficient differences were significant at the elbow ( $p$ $<0.003$ ) but, after adjustment for two comparisons, showed only a trend toward significance at the shoulder $(p=0.042)$. In the two mildly affected patients, the mean nonlinear and linear term coefficients were $0.000024 \pm 0.000010$ and $-0.0063 \pm 0.0075$, respectively, for peak shoulder and elbow angular velocities.

The reference data provided a potentially useful comparison. The normal subjects' peak shoulder angular velocities were smaller than the reference values, and their peak elbow angular velocities were greater than the reference values. The joint velocities of severely affected patients differed similarly from the reference values, but to a much greater extent (Figure 7).

The kinematics underlying the sigmoid curvature were confirmed by the finding of small positive or negative initial elbowto-shoulder angular velocity ratios (referable to the concave portions of most paths) and larger positive peak angular velocity ratios (referable to the convex portions). Severely affected patients' peak angular velocity ratios were $30 \%$ to $300 \%$ larger than normal subjects' ratios at all peak hand velocities greater

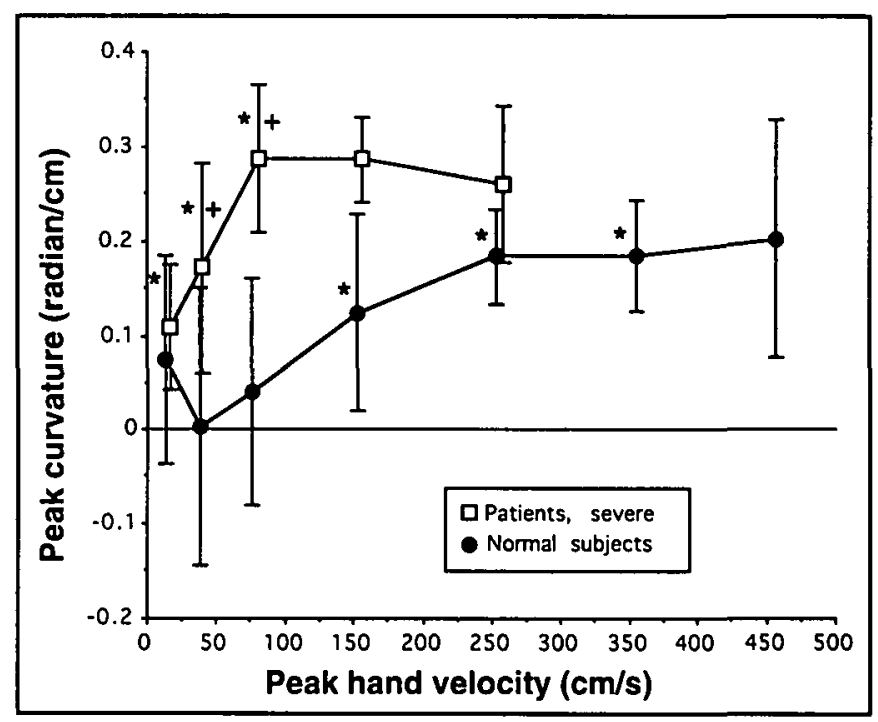

Figure 6: Peak path curvature versus peak hand velocity over the first $130 \mathrm{~ms}$ of the trajectory. Asterisks indicate that the group mean is significantly different from zero. Plus signs indicate that the group mean of patients is significantly different from the group mean of normal subjects.

than $50 \mathrm{~cm} / \mathrm{s}$, corresponding to the greater nonlinearity of their trajectories. For velocities greater than $100 \mathrm{~cm} / \mathrm{s}$, the differences were significant. Over the same hand velocity ranges, normal subjects' ratios were at least $60 \%$ larger than reference ratios.

\section{Angular Acceleration and Angular Jerk}

Overall, the mean maximal angular accelerations at both shoulder and elbow of severely affected patients were only about one-third of the mean maximal accelerations generated by normal subjects (shoulder, $49.7 \pm 10.8$ radians $/ \mathrm{s} / \mathrm{s}$ for normal subjects and $16.2 \pm 4.5$ radians $/ \mathrm{s} / \mathrm{s}$ for severely affected patients; elbow, $207.9 \pm 64.4 \mathrm{radians} / \mathrm{s} / \mathrm{s}$ for normal subjects and $70.3 \pm$ $40.6 \mathrm{radians} / \mathrm{s} / \mathrm{s}$ for severely affected patients) $(\mathrm{p}<0.0001$, shoulder; $p<0.0002$, elbow).

At the shoulder, the pattern of peak angular acceleration (Figure 8A) with respect to submaximal peak hand velocity was the same as that of peak shoulder angular velocity. Specifically, the mean nonlinear term coefficient was significantly smaller in normal subjects $(0.000020 \pm 0.000043)$ than in severely affected patients $(0.00033 \pm 0.00043)$, whereas the mean linear coefficients were comparable. This corresponds to the mild apparent plateauing of the patients' curve. Because the nonlinear coefficients were not significantly different from zero for most normal subjects and four of seven patients, simple linear regression analysis was also performed. The results showed a reduction in the mean slope of severely affected patients $(-0.08 \pm 0.03)$ compared with that of normal subjects $(-0.11 \pm 0.03)$, but the difference did not achieve significance $(\mathrm{p}<0.1)$. The reference slope $(-0.12 \pm 0.02)$ was only slightly more negative than the slope of the normal subjects.

At the elbow (Figure 8B), fits provided by either linear or second-degree polynomial regressions were less good than at the shoulder, apparently because there is very mild plateauing of patients' data and irregular rising of normal subjects' data. The overall trends captured by linear regressions, however, suggested 


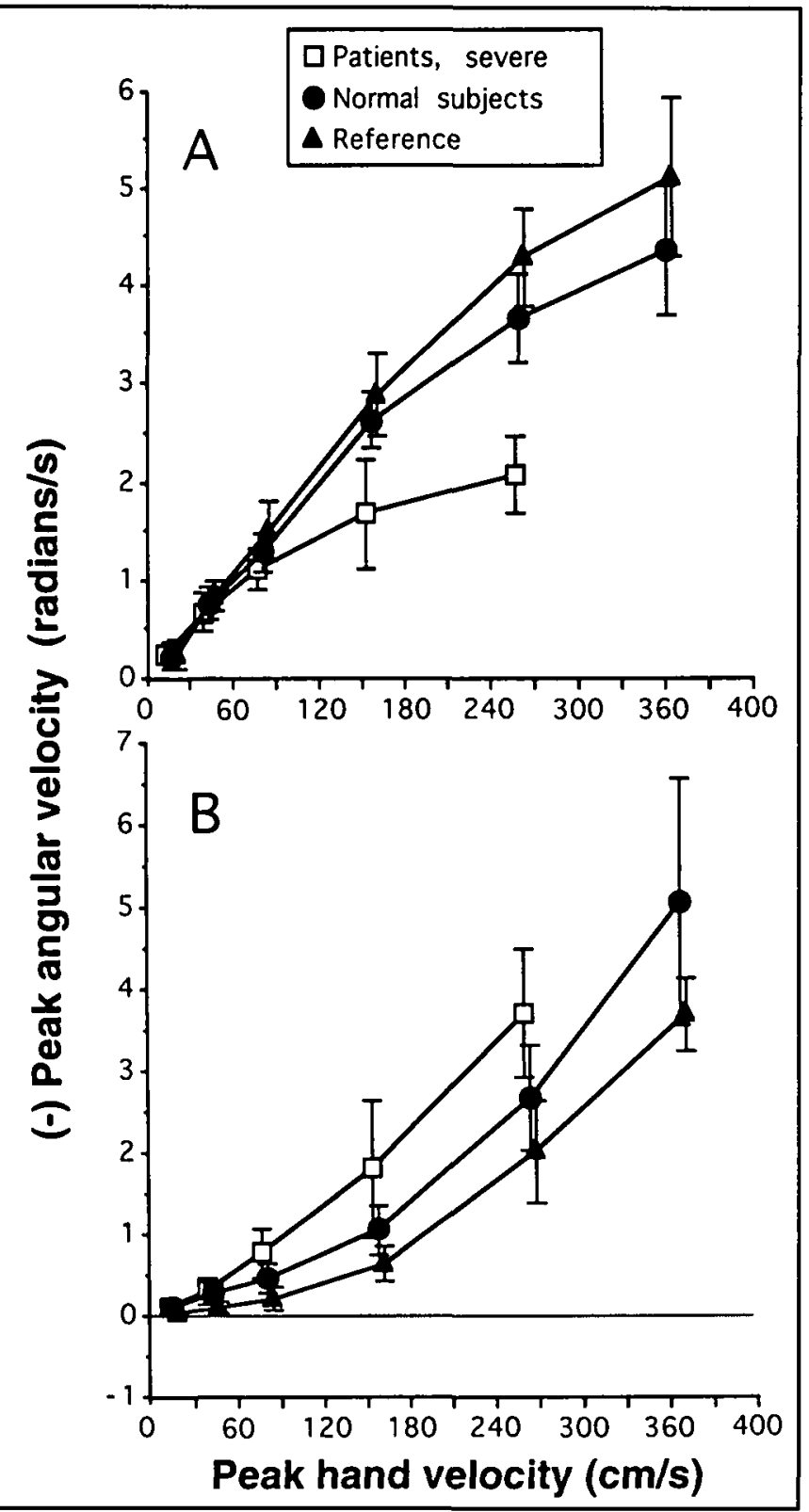

Figure 7: Negative of peak angular velocity of shoulder (A) and elbow $(B)$ versus peak hand velocity over the first $130 \mathrm{~ms}$ of movement.

that peak elbow acceleration, like shoulder acceleration but in contrast with peak elbow velocity, rose faster in normal subjects $(-0.40 \pm 0.18)$ than in severely affected patients $(-0.25 \pm 0.15)$ $(p<0.1)$. The reference slope $(-0.26 \pm 0.22)$ was between those of normal subjects and severely affected patients.

The pattern of angular jerk (not shown) was almost identical to that of angular acceleration at both joints, with the patients having subnormal jerk at the shoulder and elbow, except at low velocities, where the elbow jerk was slightly greater than that of the normal subjects.

\section{Inter-joint Interval}

The interval between the onset of elbow movement and the onset of shoulder movement was extremely "noisy," especially at velocities of less than $100 \mathrm{~cm} / \mathrm{s}$, which may account for the

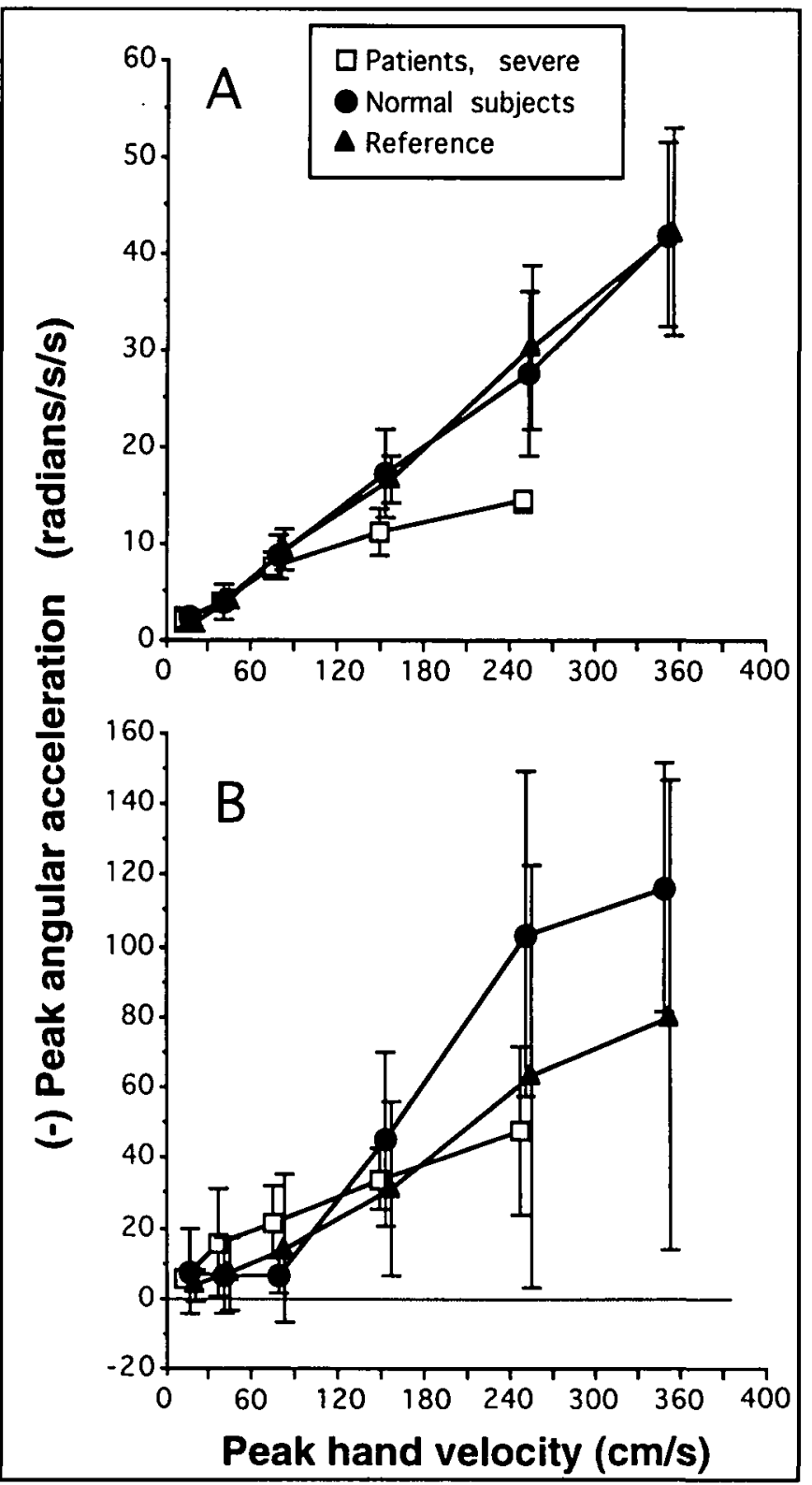

Figure 8: Negative of peak angular acceleration of shoulder $(A)$ and elbow $(B)$ versus peak hand velocity over the first $130 \mathrm{~ms}$ of movement.

absence of significant differences in the joint interval between the groups. At higher hand speeds, however, the patients showed a tendency toward intervals that were slightly shorter than the reference intervals. This is strongly consistent, physically, with the finding of above-normal peak elbow velocities in the presence of below-normal peak elbow accelerations. Compared with reference intervals, the normal subjects' intervals tended to be shorter at lower speeds and slightly longer at higher speeds.

\section{Trajectory Variability}

The variability in the subjects' trajectory measures was not analyzed extensively because the trajectory shape was strongly related to the velocity, and the subjects were asked to produce a wide range of hand speeds, as opposed to achieving specific target velocities. Therefore, the variability of the trajectory was a 
natural consequence of the task requirements and not necessarily a reflection of an inability to produce consistent results.

\section{Discussion}

\section{Systematic, Peak Velocity-Sensitive Coordination Failure During Launch}

During the very brief initial periods of the cross-body arm movements, all subjects exhibited systematic trajectory aberrations at higher peak hand velocities. These aberrations were, in fact, qualitatively the same as those that dominated much of the movement beyond $130 \mathrm{~ms}$. At higher speeds, all subjects produced sigmoidal, and in some cases nearly arcuate, paths characterized by significant curvature and, in patients, significant angular deviation as well. At lower speeds, normal subjects, and apparently also patients, had lower curvature, and both groups had little if any directional error. Thus, neither patients nor normal subjects appeared to be confused about the direction of the indicator light or to lack the desire to move directly toward it. Evidently, the path nonlinearity at higher speeds was precipitated by unavoidable failures of proper joint control in all subjects.

Associated with the characteristic path aberrations were two other important group findings. First, the maximal joint velocities and accelerations generated by the severely affected patients were significantly smaller than those generated by the normal subjects. Moreover, regressions suggested that in patients the peak angular accelerations at both joints began to saturate before maximal hand speeds were achieved. Second, velocity ratio analysis indicated that at all but the lowest hand speeds, both groups had excessive angular velocity at the elbow relative to that at the shoulder in comparison with the reference behavior. This excess elbow velocity was marked and significantly greater in the patients. Mildly affected patients almost always had kinematic findings that were intermediate between normal subjects and severely affected patients.

The ideal reference trajectories provided an important indication of the joint kinematic sources of the subjects' trajectory abnormalities. This is especially the case for the angle-angle plot (Figure 3), in which the correct joint coordination is precisely specified, independent of movement velocity, for any given geometrical relation between the subject and the instructed trajectory (dependent on arm segment lengths and relative shoulder position). However, because the calculation of the reference trajectories employed the measurable observed tangential velocity profiles in place of the unmeasurable desired profiles, the reference trajectories cannot provide the "correct" time-dependent measures (Figures 4, 7, and 8) of the angular motions in absolute terms. Still, unless the planned velocity profiles differed radically in shape from the ones observed, the reference trajectories provide a good indication of the relative joint movement appropriate for the task.

\section{Possible Primary Deficit in Rapid Torque Generation Cerebellar patients}

Because there were no important differences in the lengths of the patients' arm segments (or, by assumption, in their shapes or densities), the masses and moments of inertia of the limbs were not significantly different. Therefore, the systematic differences observed in the trajectories must reflect systematic aberrations in the applied forces. (Because the patients' arms extended slightly more than normal at the elbow during movements, their moments of inertia with respect to the shoulder increased slightly. A conservative estimate derived from a biomechanical arm mode ${ }^{22}$ indicates that this effect alone could result in at most a 5\% reduction in shoulder joint acceleration, which is considerably less than the difference we found between patients and normal subjects.)

The simplest hypothesis for explaining these data is that, as higher hand velocities were called for, patients' shoulder torques failed to keep up with those at the elbow. Hence, elbow motion began to predominate. Since, at least during launch, severely affected patients' maximal accelerations at shoulder and elbow were both substantially below normal, the mismatch between shoulder torque and elbow torque is apparently due to an especially severe deficit in shoulder torque, rather than fundamentally excessive elbow torque.

It is possible that the torque deficit in our patients resulted from reduced effort, but we doubt it for several reasons. First, the patients seemed very highly motivated to perform well. They responded well to velocity coaching at all speeds below their maximal ones, and at maximal speeds, their arms rapidly approached or overshot the table edge. Second, their peak curvatures and peak angular deviations plateaued substantially below their maximal velocities. Thus, there did not appear to be a significant speed-accuracy tradeoff at high velocities.

If we suppose that the patients' efforts were indeed comparable to those of normal subjects, we may conclude that the apparent torque deficit was due to cerebellar dysfunction. We, therefore, would hypothesize that a principal action of the cerebellum in multi-joint movements is to both facilitate and appropriately match individual joint accelerations (and decelerations), especially when large, or at least rapid, accelerations (decelerations) are required.

Our patients were not clinically weak, and several were, in fact, quite strong; the maximal forces they produced statically, against the examiner, were well within the normal range. Moreover, static or isometric weakness is neither a sign nor a symptom of chronic cerebellar disease. Mechanical principles require that if the maximal torque capacities and inertias are normal, then the overall maximal acceleration capacities must also be normal. On the other hand, the rates at which peak torque and peak acceleration are achieved need not be normal, and in our patients apparently were not. That is, the patients had absolutely reduced peak accelerations as they approached maximal hand speeds (Figures 7 and 8 ) only because peak accelerations were measured within an initial, fixed $(130 \mathrm{~ms})$ interval. We hypothesize that, in the required task, ataxia resulted from a differential reduction in the rate of torque development at the two joints. In general, torque-rate deficiency would account for the compromised performance of cerebellar patients in tasks, or parts of tasks, for which the needed changes in force level are rapid (but not necessarily large), as in, for example, high speed launches and stops, rapid alternating movements, and maintenance of upright balance.

In principle, the observed differential torque-rate impairment could be due to an inherent distal-to-proximal gradient of deficit severity in idiopathic cerebellar degeneration. Alternatively, torque-rate capacity may be globally reduced, and the deficit 
effectively greater at the shoulder simply because of this task's larger shoulder torque requirement (owing principally to the greater mass of the arm than of the forearm alone). Experiments using different torque-rate requirements would be needed to differentiate these possibilities.

Many investigators ${ }^{2,3.23}$ have postulated an important role for the cerebellum in the facilitation of movement rate. The idea is consistent with the adjustable pattern-generator model proposed by Houk et al., ${ }^{24}$ which suggests that an important function of the cerebellum is to facilitate the rapid spread of neural excitation to the primary motor cortex in response to pre-motor trigger signals. It is also consistent with the observations of Mai et al. ${ }^{25}$ that, even in the absence of movement, patients with cerebellar degeneration and normal strength demonstrated isometric forcerate deficits. Moreover, the severity of the finger force-rate impairment correlated roughly with the severity of clinical dysdiadochokinesia in the hand. ${ }^{25}$ (Table and Fig. 9B) Most importantly, the hypothesis of generalized torque-rate deficiency provides a connection between the reduced acceleration occurring in multijoint ataxia ${ }^{6.11}$ and the reduced angular acceleration associated with overshoot in single-joint movements. ${ }^{2-5,7-9}$

The hypothesis also raises the possibility that abnormalities of the inter-joint interval within some ataxic movements occur as the, often minor, consequence of altered joint accelerations and not as primary defects. Thus, the trend toward decreased elbow latency in our study may have occurred simply because the shoulder was more sluggish than the elbow. A study of multi-joint ball throwing in cerebellar patients by Becker et al. ${ }^{12}$ similarly reveals torque-rate deficiencies. Measured kinematic intervals were also not significantly different from those of normal subjects. However, there was a trend toward decreased latency between the onset of hand opening and the peak of distal forearm velocity that followed. As in our task, this tendency may be attributed to a relative sluggishness in the development of the first action. This is very plausible because hand opening during throwing requires particularly rapid joint acceleration.

On the other hand, Spidalieri et al. ${ }^{26}$ noted no apparent change in buildup on the EMG or slowing of acceleration in single-joint arm movements in monkeys with cerebellar lesions. This study, however, involved unilateral lesions affecting principally the dentate nucleus, whereas the aforementioned studies in humans included subjects with cerebellar dysfunction that likely affected circuits traversing most, if not all, deep cerebellar nuclei bilaterally. The results of Spidalieri et al. ${ }^{26}$ are consistent with those of Meyer-Lohmann et al., ${ }^{3}$ who found no acceleration abnormality when single cooling probes were inserted either laterally or medially to the dentate, and it is not evident that the monkeys were ataxic. However, Meyer-Lohmann et al., ${ }^{3}$ found diminished initial velocity when both probes were inserted simultaneously (a condition under which both dentate and interpositus are likely to be more severely compromised). We infer that this was associated with ataxia, as both Miall et al. ${ }^{27}$ and Hore and Flament ${ }^{28}$ described ataxia when cooling probes were inserted where both the interpositus and dentate (or dentatothalamic fibers) could be affected. Similarly, Goodkin et al. ${ }^{11}$ described no significant change in acceleration of a patient's brief, nonvisually guided wrist movements after a unilateral cerebellar stroke, despite persistent abnormalities in longer, visually guided, compound multi-joint arm movements. The stroke, involving the left superior cerebellar artery, had appar- ently damaged mainly the ipsilateral dentate nucleus and portions, but not all, of the cerebellar cortex projecting to the interpositus and fastigius. The findings were obtained more than a year postictally and after considerable occupational therapy. We consider that the normal acceleration may reflect the development, over many months, of compensation by the surviving cortex and nuclei, as may occur in recovery from unilateral dentate lesions in monkeys. ${ }^{29}$ In support of this possibility are the ataxia and corresponding decreased initial acceleration in single-joint arm movements of a patient studied by Brown et al. ${ }^{6}$ (Table 1 and Fig.1) soon after a stroke that also involved the superior cerebellar artery.

Finally, Thach et al. ${ }^{10}$ highlighted the neuroanatomical and neurophysiological evidence for the critical role of the cerebellum in coordination between joints. We, therefore, emphasize that our hypothesis does not imply that control of each individual joint within a multi-joint movement occurs in isolation. In fact, it is quite plausible, and consistent with our findings, that the cerebellum normally "calculates" the amount of muscle activation appropriate for each joint on the basis of local and, when present, multi-joint dynamic requirements.

\section{Normal subjects}

The curved trajectories generated by normal subjects at very high speeds may also be understandable from the perspective of torque-rate impairment, but the argument is slightly more complex because the deficit is much milder, and potential compensation strategies are likely to be more flexible. Normal subjects also had decreased peak shoulder velocities relative to the reference velocities, but to a much smaller degree than in patients. Unlike in patients, however, at very high velocities, their interjoint latencies, elbow accelerations, and elbow jerks tended to be greater than the reference values. If at very high speeds normal subjects began to encounter mildly greater sluggishness at the shoulder than at the elbow, their hands would have tended to project centrifugally, beyond the target direction, as with the patients. One of two simple compensatory strategies to improve directional accuracy might then be invoked. The elbow velocity could be reduced to better match the actual shoulder velocity, but this would also reduce the peak hand velocity and hence be contrary to the instructions. Alternatively, the onset of elbow extension could be delayed and perhaps preceded by a slight flexion (which might well be a passive movement due to reaction forces) while the elbow's late acceleration is maintained, or even augmented, in a whip-like maneuver. The tactic more effectively preserves hand speed and correct final direction, but yields an early, mild inward trajectory deviation consistent with our observations. Since the tactic relies on a substantially preserved capacity to generate rapid elbow accelerations, it is an option that is presumably unavailable to severely affected cerebellar patients.

\section{Alternative Explanations: Primary Derangements in Timing}

Although difficulty with torque generation is apparently an important problem in cerebellar patients, it may not be the only or even the primary mechanism underlying the trajectory abnormalities. Patients may be fundamentally limited in their ability to time small intervals correctly. ${ }^{30,31}$ Although a preferred direction for the misjudgment was not reported, ${ }^{30}$ perhaps our patients tended to underestimate the correct inter-joint interval, 
which caused premature and, therefore, relatively excessive action at the elbow. This principle, while not explaining why patients' maximal-effort shoulder accelerations should be deficient, might explain why they rarely generated prolonged interjoint intervals to compensate for the excessive elbow velocities, as we suggest normal subjects did. Thus, it may be for this reason that patients were less able to correct their directional errors.

Perhaps a central abnormality in cerebellar ataxia is simply an inappropriate degree of synchrony in joint actions. Militating against this hypothesis is the common clinical observation of movement decomposition in cerebellar patients. ${ }^{2}$ This phenomenon has been described by Goodkin et al.," who showed that their cerebellar stroke patient had inappropriate joint asynchrony in a multi-joint reaching task. As the authors suggested, the patient may have employed asynchronous joint movements strategically to improve targeting accuracy. A more specific interpretation of the initial movement pattern is that initial elbow relaxation was used as compensation for impaired torque rate at the shoulder. On the affected side, there was both reduced initial angular acceleration at the shoulder and reduced (in fact, negative) initial angular acceleration at the elbow relative to the shoulder. ${ }^{11}$ (Figs. $4 \mathrm{C}$ and $5 \mathrm{C}$ ) Because the reach was against gravity, normal performance required considerable initial torque-rate at the shoulder, but reduction or reversal of elbow acceleration diminished the requirement. This strategy was acceptable in their task because end point accuracy, rather than speed or precise path linearity, was the principal objective.

\section{A Selected Component of Cerebellar Control}

Our severely affected patients had cerebellar degenerations that caused midline and appendicular ataxia. Hence, it is likely that many, if not all, of their cerebellar anatomical subsystems were abnormal. Moreover, the patients with olivopontocerebellar atrophy presumably also had some degeneration of cerebellar input nuclei. Thus, we interpret our results as reflecting the dysfunction of central trans-cerebellar circuits without attempting to differentiate between abnormalities of the pre-cerebellar nuclei and intracerebellar components. Because this experiment was designed deliberately to avoid the effects of conscious feedback control loops, it potentially did not evaluate some important aspects of cerebellar operation. On the basis of neural signal transmission times, we expect that our paradigm largely excluded effects due to peripherally triggered, dentate-dependent cerebro-cerebello-corticospinal feedback, which participates in consciously controlled, direction-sensitive responses to limb perturbation. ${ }^{18,32}$ Instead, our results appear to be primarily due to the behavior of shorter trans-cerebellar feedforward and trans-interpositus or trans-fastigius feedback pathways. This would not apply to the path deviation values for the slow movements occurring beyond the $130-\mathrm{ms}$ limit. Some of these measurements might reflect contributions from dentate-dependent feedback pathways. On the other hand, in virtually all of the movements studied, the kinematic patterns identifiable within the first $130 \mathrm{~ms}$ continued to be present for hundreds of milliseconds longer. Thus, in this task, it is possible that much, if not most, of the movement was stabilized principally by cerebellar circuits other than those that specifically mediate conscious error correction.

Our findings indicate that lesions affecting some or all of the shorter trans-cerebellar pathways are sufficient to produce a sys- tematic abnormality of the trajectory in a multi-joint movement, which appears to result from torque-rate impairments of differing severity at different joints. Because decreased torque-rate is also observed in single-joint movements, it may be an underlying characteristic of ataxic movements associated with generalized cerebellar dysfunction. Confirmation of this hypothesis would require detailed analysis of the kinetics of this and other movements. Certainly, the proposed mechanism may be insufficient to account for all abnormalities of multi-joint coordination caused by cerebellar lesions, particularly in movements involving conscious feedback, in which further cerebellar circuits participate in movement control.

\section{ACKNOWLEDGEMENTS}

We thank Dr. Thomas A. Zeffiro for assistance with data collection hardware and software, Dr. Lisa McShane for statistical consultation, and B.J. Hessie for skillful editing.

\section{REFERENCES}

1. Liu CN, Chambers WW. A study of cerebellar dyskinesia in bilaterally deafferented forelimbs of the monkey. Acta Neurobiol Exp 1971; 31: 263-289.

2. Holmes G. The cerebellum of man. Brain 1939; 62:1-30.

3. Meyer-Lohmann J, Hore J, Brooks VB. Cerebellar participation in generation of prompt arm movements. J Neurophysiol 1977; 40: 1038-1050.

4. Hallett M, Shahani BT, Young RR. EMG analysis of patients with cerebellar deficits. J Neurol Neurosurg Psychiatry 1975; 38: 1163-1169.

5. Hallett M, Berardelli A, Matheson J, Rothwell J, Marsden CD. Physiological analysis of simple rapid movements in patients with cerebellar deficits. J Neurol Neurosurg Psychiatry 1991; 53: 24-133.

6. Brown SH, Hefter H, Mertens M, Freund H-J. Disturbances in human arm movement trajectory due to mild cerebellar dysfunction. J Neurol Neurosurg Psychiatry 1990; 53: 306-313.

7. Hore J, Wild B, Diener H-C. Cerebellar dysmetria at the elbow, wrist and fingers. J Neurophysiol 1991; 65: 563-571.

8. Flament D, Hore J. Movement and electromyographic disorders associated with cerebellar dysmetria. J Neurophysiol 1986; 55: $1221-1233$.

9. Manto M, Godaux E, Jacquy J. Cerebellar hypermetria is larger when inertial load is artificially increased. Ann Neurol 1994; 35: 45-52.

10. Thach WT, Goodkin HP, Keating JG. The cerebellum and the adaptive coordination of movement. Annu Rev Neurosci 1992; 15: 402-403.

11. Goodkin HP, Keating JG, Martin TA, Thach WT. Preserved simple and impaired compound movement after infarction in the territory of the superior cerebellar artery. Can J Neurol Sci 1993; 20 (Suppl 3): S93-S104.

12. Becker WJ, Kunesch E, Freund H. Coordination of a multi-joint movement in normal humans and in patients with cerebellar dysfunction. Can J Neurol Sci 1990; 17: 264-274.

13. Gilman S, Carr D, Hollenberg J. Kinematic effects of deafferentation and cerebellar ablation. Brain 1976; 99: 311-330.

14. Kane SA, Goodkin HP, Keating JG, Thach WT. Incoordination in attempted reaching and pinching after inactivation of cerebellar dentate nucleus. Soc Neurosci Abstr 1989; 15: 52.

15. Fitts PM. The information capacity of the human motor system in controlling the amplitude of movement. J Exp Psychol 1954; 47: 381-391.

16. Miller RG, Freund H-J. Cerebellar dyssynergia in humans - a quantitative analysis. Ann Neurol 1980; 8: 574-579.

17. Sanes JN, Lewitt PA, Mauritz K-A. Visual and mechanical control of postural and kinetic tremor in cerbellar system disorders. J Neurol Neurosurg Psychiatry 1988; 51 : 934-943. 
18. Crago PE, Houk JC, Hasan Z. Regulatory actions of human stretch reflex. J Neurophysiol 1976; 39: 925-35.

19. Favilla M, Hening W, Ghez C. Trajectory control in targeted force impulses. Exp Brain Res 1989; 75: 280-294.

20. Snedecor GW, Cochran WG. Statistical Methods, 7 th edition. Ames, lowa: Iowa State University Press, 1980: 1 16, 274-297.

21. Flash T. The control of hand equilibrium trajectories in multi-joint arm movements. Biol Cybern 1987; 57: 257-274.

22. Uno $Y$, Kawato $M$, Suzuki R. Formation and control of optimal trajectory in human multijoint arm movement. Biol Cybern 1989; 61: 89-10.

23. Meyer-Lohmann J, Conrad B, Matsunami K, Brooks VB. Effects of dentate cooling on precentral unit activity following torque pulse injections into elbow movements. Brain Res 1975; 94: 237-251.

24. Houk JC, Keifer J, Barto AG. Distributed motor commands in the limb premotor network. Trends Neurosci 1993; 16: 27-33.

25. Mai N, Bolsinger P, Avarello M, Diener H-C, Dichgans J. Control of isometric finger force in patients with cerebellar disease. Brain 1988; 111: 973-998.

26. Spidalieri G, Busby L, Lamarre Y. Fast ballistic arm movements triggered by visual and somesthetic stimuli in the monkey. II.
Effects of unilateral dentate lesion on discharge of precentral cortical neurons and reaction. J Neurophysiol 1983; 50: 1359 . 1379.

27. Miall RC, Weir DJ, Stein JF. Visuomotor tracking during reversible inactivation of the cerebellum. Exp Brain Res 1981; 65: 455464.

28. Hore J, Flament D. Evidence that a disordered servo-like mechanism contributes to tremor in movements during cerebellar dysfunction. J Neurophysiol 1986; 56: 123-136.

29. Trouche E, Beaubatron D. Initiation of a goal directed movement in the monkey. The role of the cerebellar dentate nucleus. Exp Brain Res 1980; 40: 311-321.

30. Keele SW, Ivry R. Does the cerebellum provide a common computation for diverse tasks? A timing hypothesis. Ann NY Acad Sci 1990; 608: 179-207; discussion 207-211.

31. Grill SE, Hallett M, Marcus C, McShane L. Disturbances of kinesthesia in patients with cerebellar disorders. Brain 1994; 117 , 1433-1447.

32. Strick PL. The influence of motor preparation on the response of cerebellar neurons to limb displacements. J Neurosci 1983; 3: 2007-2020. 